\title{
FLORIDO DEL CORRAL, DAVID (2010). El espacio portuario de Conil. Infraestructuras socioeconómicas, políticas y de ingeniería. Sevilla: Agencia Pública de Puertos de Andalucía, 298 pp.
}

\section{Juan Antonio Rubio-Ardanaz \\ Universidad de Extremadura}

El puerto como realidad resultante de una serie de procesos que tienen lugar en un punto de la costa andaluza implicando un colectivo de hombres y mujeres fundamentalmente ligados al trabajo pescador. David Florido del Corral, toma como punto de partida este parámetro para centrarse en el Puerto de Cabo Roche en Conil (Cádiz, Andalucía), superando cualquier visión estática o meramente descriptiva y aborda el estudio del espacio portuario y su consolidación, en tanto que ligados a realidades sociales con una historia y un devenir económico que para este antropólogo es preciso explicar en profundidad.

Con esta intención va a cubrir y completar un vacío importante, existente hasta la fecha en el campo de los estudios sociales y más en concreto de la antropología marítima, sentando una premisa fundamental al entender que el espacio portuario comprende y es un conjunto de procesos, sistemas de relaciones y actividades sociales. Desde esta orientación dirigirá la reflexión al campo de lo social, dando entrada a una visión antropológica con la cual poder superar, profundizando y en su caso complementando los niveles propios del ámbito de la planificación técnica de los usos y de la gestión portuaria. En este sentido es importante por lo tanto, tener en cuenta la relevancia y consecuencias de las dinámicas socioeconómicas y culturales que irán apareciendo cronológicamente sobre la escena portuaria y pesquera de la localidad estudiada.

En el plano teórico, se da respuesta a una línea analítica que parte de la necesidad de clarificar y mostrar las relaciones de los colectivos pescadores con el marco socioeconómico, político y ecológico. Para ello ha sido primordial una orientación 
que toma en consideración la perspectiva global/local y que se traduce en una visión de la historia como resultado de la articulación compleja, dinámica y conflictiva de los distintos procesos, tanto endógenos como exógenos, locales y globales. En este sentido, estudiar el marco histórico del puerto de Conil y su colectivo más representativo, el de los pescadores artesanales, exige incidir en la importancia de la trama de relaciones tanto locales como globales.

Metodológicamente contamos con un modelo que se muestra eficaz y resolutivo a la hora de obtener los datos suficientes con los que ir hilando el entramado de las bases socioculturales que están en la base del surgimiento de este nuevo puerto, cuyo análisis conduce a hablar necesariamente con y de hombres y mujeres concretos, con sus organizaciones representativas propias como la Cofradía de Pescadores (o la Sociedad de Obreros Pescadores y el Pósito de Pescadores, anteriores a ésta), vehículo fundamental a la hora de reivindicar el complejo infraestructural con el que poder garantizar la continuidad histórica y la reproducción social del colectivo pescador. Ha sido necesario acceder y obtener los datos que muestran sus acciones corporativas, sus estrategias económicas y políticas, así como sus líneas de actuación. Las explicaciones han exigido un amplio y detallado conjunto de referencias articuladas en el marco institucional y de la economía política, correspondientes a los períodos estudiados (primer tercio del siglo XX, dictadura franquista, y época autonómica hasta la actualidad). Ha sido preciso mostrar cómo el Estado, institucionalmente hablando, llega a modelar e influir en los comportamientos económicos y políticos de los pescadores. Y también cómo éstos, organizadamente y apoyándose en sus instituciones, han desarrollado comportamientos localizados, en función de valores, prácticas y expectativas de base vernácula para adaptarse o bien rechazar el marco político y económico, tanto de procedencia global como de origen cultural local.

Esta eficacia y resolución metodológicas se han alcanzado recurriendo a las labores de trabajo de campo propias de la disciplina antropológica, así como a una extensa búsqueda documental que aparece, tanto narrativamente (destacando al respecto libros de actas, libros de pólizas de seguros, subsidio familiar, actas capitulares del Ayuntamiento, libros de correspondencia, etc.), como vertida ordenadamente a lo largo de las páginas del libro por medio de cuadros, mapas, gráficos e ilustraciones (significativos dibujos obtenidos sobre el terreno y fotografías puntuales). En lo que se refiere al trabajo sobre el terreno, el autor señala y reconoce de una manera muy clarificadora y que es de agradecer, la importancia que han tenido las "entrevistas en profundidad", fuente de información cualitativa procedente directamente de los protagonistas de las dinámicas económicas, políticas y sociales en su conjunto. Por otro lado y en lo que toca al terreno documental, David Florido del Corral destaca también la importancia que ha supuesto para la investigación, el acceso a los proyectos de ingeniería portuaria de Cabo Roche y a otros 
proyectos e informaciones de tipo técnico, concretamente en el Archivo del Puerto de Barbate cuyos datos fundamentan buena parte de su investigación. A todo ello, se suman el recurso a obras de temática referida al cambio desde formas de pesca artesanales a formas industriales, así como a la extensa producción legislativa correspondiente a las épocas históricas tratadas y analizadas.

Estos planteamientos, teóricoymetodológico, como hemospodidocomprobar dispondrán y capacitarán al autor para elaborar tres capítulos fundamentales intensamente revestido de una amplia etnografía y de datos explicativos. En ellos analiza pormenorizadamente y desarrolla cronológicamente, primero cada uno de los períodos históricos del pasado reciente, deteniéndose y remarcando las bases de la economía pesquera y sus avatares, la incidencia de las políticas portuarias y pesqueras, la evolución del sector y los proyectos portuarios, correspondientes al primer tercio del siglo XX y a la dictadura subsiguiente (capítulos primero y segundo respectivamente). A partir de aquí y en segundo lugar, se detiene en el período más cercano en el tiempo (desde el inicio de la etapa política autonómica a la actualidad), caracterizado por una consolidación de la economía pesquera en un espacio portuario en el que ahora tienen lugar trabajos permanentes de comercialización del pescado y se construyen y reparan embarcaciones, implicando un conjunto de entidades empresariales vinculadas con la economía pesquera.

Esta viva dinámica de la actividad portuaria y pesquera local van a ser prueba de la “artesanalización”, concepto de alto interés y que representa en palabras de David Florido del Corral, el "fortalecimiento económico, social y político de las flotas de menores dimensiones y con una organización socioeconómica predominantemente familiar" ( $p$. 141). La construcción del puerto pesquero en Cabo Roche, además de la vigorización del sector durante este período, (nos referimos al más reciente y actual), será primordial para el desarrollo de las estrategias económicas tanto de los pescadores, como de otros agentes económicos dispuestos a ejercer el control y la gestión desde el ámbito de la política local. Las iniciativas de la administración a favor del sector pesquero se van a revelar además como fuente de interesantes resultados socioeconómicos a nivel local.

En una primera síntesis, tenemos que confirmar que la antropología marítima en la perspectiva planteada por el autor, se nos brinda como una oportunidad recurrente para comprender en profundidad qué ha sucedido y qué está sucediendo en el ámbito de la pesca a nivel local y en correlación con la esfera global. En este sentido, ha sido capaz de correlacionar la importancia de la construcción de una obra de ingeniería portuaria como la llevada a efecto en Cabo Roche y las dinámicas políticas, económicas y sociales analizadas que han incidido a nivel microsocial, en un proceso permanente de optimización de las infraestructuras. El estudio nos da las bases suficientes para comprender que de este modo se conseguirá desarrollar actividades de pesca que de otra manera no hubieran sido posibles, atrayendo al capital, poniendo en marcha iniciativas 
empresariales y dando las bases suficientes para el mantenimiento de familias pescadoras integradas en la amplia estructura socioeconómica de Conil de la Frontera.

Junto a esta aportación, también ha quedado claro que si bien las iniciativas portuarias por parte de la administración plantean muy frecuentemente limitaciones importantes, éstas pueden dar lugar a procesos socioeconómicos dinámicos y vivos. A pesar de las frustraciones históricas descritas y vividas por los pescadores, primero frente a la administración central durante el primer tercio del siglo pasado y luego ante las constantes negativas durante la administración de la dictadura franquista, llegará un momento en el que tiene lugar una clara acción propiciadora por parte estatal, tanto central como comunitaria y andaluza. Esto desembocará en un proceso abierto en el que se llega actualmente a conformar un espacio portuario esencial para la pesca artesanal que incluso en un futuro puede albergar actividades náuticas deportivas de envergadura, además de otras relacionadas con la acuicultura y con otro tipo de innovaciones como el acceso a nuevas licencias de pesca durante los últimos años, a lo que se suman otras importantes iniciativas relacionadas con la mejora de la comercialización del pescado. En esta dirección, David Florido del Corral nos clarifica la dinámica histórica y estructural que ha conducido a la actual situación, en nuestra opinión a esta nueva maritimidad que se configura en nuestros puertos pesqueros a múltiples niveles y a cuya explicación es preciso dar cauces de conocimiento.

Terminaremos indicando la pertinencia de la construcción histórica realizada por David Florido del Corral que nos ha permitido comprender la importancia de la actividad estatal y del papel y acciones llevadas a cabo por parte de los agentes sociales de cara a su optimización y definición continua. Como ya hemos indicado, al respecto han incidido de forma fundamental las labores de la Cofradía de Pescadores, empeñada en la mejora de los servicios pesqueros, controlando la actividad, comercialización, ordenamiento de la flota y gestión de los recursos. En el marco de esta dinámica procesual conducente a la reforma del espacio portuario se prevé el incremento de la actividad náutico-deportiva como ya se ha indicado. Y acabaremos también incidiendo en la superación que comporta este trabajo de investigación y su planteamiento, más allá de las previsiones y alcance realizados desde el plano técnico, referentes sobre todo a la dimensión social. Los proyectos técnicos auguraban la transformación de las estructuras pesqueras locales hacia su industrialización, premonición que no se cumplirá al mantenerse dentro del modelo artesanal. Tal como se muestra a lo largo del trabajo, es preciso considerar otras variables de carácter cualitativo, relacionadas con estrategias de tipo productivo y político de los armadores, sus representantes, autoridades locales, así como los proyectos de política sectorial y regional. En palabras del autor, las actividades económicas, sociales y políticas "son desarrolladas por agentes sociales, cuyas perspectivas culturales modifican y crean condiciones de producción social que no se pueden prever a partir de ecuaciones 
matemáticas" (p. 283). Como vemos, la mecanicidad de esta clase de proyecciones no es suficiente, siendo preciso introducir en la planificación y en su posterior análisis, las perspectivas de los usuarios, su lógica cultural, es decir "su modo de hacer y de pensar el mundo" (p.284).

La realización del puerto y su progresiva ampliación y mejora han servido para consolidar el sector pesquero de Conil en cuya base social aparecen familias de tradición pesquera local. Éstas han entrado en una dinámica de transformación de la actividad pesquera aprovechando las oportunidades ofrecidas por el nuevo contexto de economía política. Sin embargo, emergen mercados de trabajo concurrentes, más tentadores y accesibles. Aquí aparecen otras situaciones, en nuestras palabras otra vez, esa nueva maritimidad, y en palabras de David Florido del Corral posibles factores que pueden ser decisivos y que pueden ir alternando la racionalidad cultural basada en la pesca. Este sería uno de los retos para seguir investigando en el campo antropológico marítimo. De todas formas, ha quedado meritoria y explícitamente claro por parte del autor que una perspectiva capaz de articular procesos y dinámicas locales y globales, para entender mejor cómo se va produciendo la historia de cualquier fenómeno social, es pertinentemente posible. 


\section{REFERENCIAS BIBLIOGRÁFICAS}

Florido del Corral, D. (2002) Un siglo de política e instituciones pesqueras en Andalucía. Sevilla: Consejería de Agricultura y Pesca de la Junta de Andalucía, Fundación Blas Infante.

Florido del Corral, D. (2002) "Políticas globales y desigualdades locales: efectos socioeconómicos de la política pesquera comunitaria". Revista de Estudios Regionales, 62, pp. 79-119.

Florido del Corral, D. (2003) "Las flotas artesanales andaluzas en la era de la 'globalización'. Desafíos teóricos y prácticos de un proceso conflictivo". En J. A. Rubio-Ardanaz (ed.) La pesca y el mar: cambio sociocultural y económico. Zainak, Cuadernos de AntropologíaEtnografía, 25. San Sebastián: Sociedad de Estudios Vascos, pp. 195-216.

Rubio-Ardanzaz, J. A. (2003) "Presentación. Las realidades marítimo-costeras como expresión de maritimidad y objeto de estudio antropológico". En J. A. Rubio-Ardanaz (ed.) La antropología marítima y el crisol de la maritimidad: profesiones, economías, normativas, patrimonio y símbolos. Zainak, Cuadernos de Antropología-Etnografía, 33. San Sebastián: Sociedad de Estudios Vascos, pp. 195-216. 